\title{
Downregulation of Hsa-miR-3616-3p in Triple-Negative Breast Cancer is Associated with Cell Migration and Invasion
}

Keqian Wu

Chongqing Medical University https://orcid.org/0000-0002-4113-0296

Rui Peng

Chongqing Medical University

Zheng Zhang

Chongqing Medical University

Handeng Liu

Chongqing Medical University

Yan Sun ( $\square$ yansun@cqmu.edu.cn )

Chongqing Medical University

Research

Keywords: hsa-miR-3616-3p, triple-negative breast cancer, bioinformatics analysis

Posted Date: November 1st, 2021

DOI: https://doi.org/10.21203/rs.3.rs-824447/v1

License: (c) (1) This work is licensed under a Creative Commons Attribution 4.0 International License. Read Full License 


\section{Abstract}

Background: Micro RNA (miRNA), a class of endogenous small RNAs with a length of about 20-24 nucleotides, have been played a variety of important regulatory roles in cells and have attracted the attention of researchers because involvement of initiation and progression of diverse kinds of human diseases, especially cancer. However, the regulatory role of miRNA in triple negative breast cancer (TNBC), its clinical significance and potential mechanism are still largely unknown.

Methods: In this study, the differentially expressed miRNAs were analyzed using GEO2R from GSE57897 and GSE97811 of the Gene Expression Omnibus (GEO). Then, we performed the overall survival (OS) analysis of four candidate miRNAs. And the expressions of four candidate miRNAs in TNBC and non-TNBC tissues were tested by Quantitative real-time PCR. We determined the level and prognostic values of hsa-miR-3616-3p in TNBC patients. Then, TNBC cells migration and invasion were studied in vitro with hsa-miR-3616-3p by using wounding heal assays and transwell assays. Next, target genes and transcription factors of hsa-miR-3616-3p enrichment analysis, were performed by the Gene Ontology (GO), Kyoto Encyclopedia of Genes and Genomes (KEGG) pathway.

Results: Based on GSE57897 and GSE97811, we found 962 differentially expressed miRNAs including 943 up-regulated miRNAs and 19 down-regulated miRNAs. We selected top2 up-regulated miRNAs as hsa-miR-4428 and hsa-miR-575, and top2 down-regulated miRNAs as hsa-miR-3616-3p and hsa-miR-3909, and then we found that low expression of 4 candidated miRNAs had a worse prognosis in TNBC subtype. Next, we verified that the expression of hsa-miR-3616-3p was the most downregulated in TNBC tissues compared with matched surrounding tissues by qRT-PCR. Moerover, our results showed that overexpression of hsa-miR-3616-3p inhibited TNBC cells migration, and invasion in vitro.

Conclusions: Our study might reveal important roles of hsa-miR-3616-3p plays in TNBC migration and invasion.

\section{Introduction}

Breast cancer (BC) is one of the most popular malignant tumors among women and the second major cause of cancer-related deaths in women worldwide [1]. TNBC accounts for $15 \% \sim 20 \%$ of all the pathological types of breast cancer without expression of estrogen receptor (ER), progesterone receptor (PR) and human epidermal growth factor receptor2 (HER2) [2]. It has special biological behavior and clinicopathological characteristics. Compared with other breast cancer subtypes, TNBC occurs more frequently in young women, usually with a high histologic grade and poor prognosis[3]. The most important characteristics of TNBC are high proliferation and frequent metastasis to the lung and brain[4]. Although decades of research have provided considerable insight into the multistep transfer process, little is known about the molecular basis of TNBC transfer. The main therapeutic strategies are conventional chemotherapy and radiation. To date, no targeted therapy for TNBC has been approved by Food and Drug Administration (FDA) [5, 6]. Therefore, it is urgent to find out prognosis predictive factors and potential therapeutic targets in BC diagnosis and treatment. And, further elucidation of the molecular mechanisms of TNBC metastasis is essential for the development of new treatments and the successful treatment of TNBC patients.

MiRNA is a highly conserved non-coding small RNA, of 21-23 nucleotides that can regulate gene expression at the post-transcriptional level by inhibiting protein translation or degradation of target mRNA[7]. As we all know, microRNAs can serve as either an oncogene or a tumor suppressor in the occurrence and development of various cancers, including breast cancer[8,9]. Some miRNAs were also found to regulate cancer cell growth and metastasis in lung cancer, liver cancer, colorectal cancer, gastric cancer, renal cancer, prostate cancer,breast cancer and other types of cancer[10-16]. To date, the functions of only a few miRNAs have been well characterized, and the biological functions of most miRNAs remain unknown.

In our study, the differentially expressed miRNAs were analyzed using GEO2R from GSE57897 and GSE97811 of the Gene Expression Omnibus (GEO). We found 4 candidate miRNAs and performed the overall survival (OS) analysis. The results showed that a high expression of hsa-miR-4428, hsa-miR-575, hsa-miR-3909 and hsa-miR-3616-3p were associated with poor prognosis in the BC, but great prognosis in the TNBC. Therefore, we further verified the relationship between 4 candidate miRNAs and TNBC, we detected the expression of four candidate miRNAs in 15 TNBC tissues and 15 non-TNBC tissues by using real-time qPCR. Our qPCR data showed that the expressions of hsa-miR-575, hsa-miR-3909 and hsa-miR-3616-3p were consistent with the result of the microarray data and hsa-miR3616-3p was the most significant and stable down-regulation in TNBC tissues. Hsa-miR-3616-3p overexpression inhibited migration and invasion of TNBC cells in vitro. We found that target genes and transcription factors of hsa-miR-3616-3p were associated with TNBC. Our work may reveal the important role of hsa-miR-3616-3p in the occurrence and development of TNBC, and hsa-miR-3616-3p may be clinically valuable biomarkers and novel therapeutic targets. 


\section{Materials And Methods}

\subsection{Microarray data.}

The Gene Expression Omnibus database (http://www.ncbi.nlm.nih.gov/geo) is a public genome database[17]. In this study, two Noncoding RNA profiles GSE57897 and GSE97811 were downloaded from the GEO. The criteria for both Non-coding RNA profiles were (a) the samples included two groups of breast cancer tissue samples and normal breast tissue samples; (b) the sample size of each Non-coding RNA profile was greater than 60; (c) they were recently updated in the last two years (2019-2020). The GSE57897 and GSE97811 were derived from GPL18722 and GPL21263 platform, respectively. The GSE57897 data profile contained 422 breast cancer tissue samples and 31 normal breast tissue samples. The GSE97811 data profile contained 45 breast cancer tissue samples and 16 normal breast tissue samples.

\subsection{Identification of differentially expressed miRNAs}

GEO2R (https://www.ncbi.nlm.nih.gov/geo/geo2r/) online analysis software was used to analyze the differentially expressed miRNAs in breast cancer tissue samples and normal breast tissue samples. They were analyzed by Limma rapid differential analysis tool in sanger box (http://sangerbox.com/). $p<0.05$ was used as the cut-off criteria of differentially expressed miRNAs.

\subsection{Survival analysis of candidate miRNAs}

The OS analysis of 4 candidate miRNAs was performed using the online Kaplan-Meier Plotter survival analysis server (https://kmplot.com/analysis/). The $p<0.05$ was considered to indicate statistical significance, and screened out promising miRNAs with the prognostic value.

\subsection{Tissue samples}

The samples of 15 TNBC tissues and 15 non- TNBC tissues were from patients in the First Affiliated Hospital of Chongqing Medical University (Chongqing, China). Tissue samples of patients were treated without chemotherapy or radiotherapy before surgical resection and all patients gave informed consent. The tissue samples were quickly cryopreserved with liquid nitrogen tanks for further analysis. All biopsy tissue samples of patients were diagnosed as TNBC or non-TNBC by immunohistochemical staining. The study was conducted in accordance with the Helsinki Declaration and has been approved by the Ethics Committee of Chongqing Medical University.

\subsection{RNA extraction and the qRT-PCR analysis of miRNAs expression}

Total RNA of TNBC or non-TNBC tissue samples and TNBC cells was extracted by RNAiso Reagent (TaKaRa, China) under the manufacturer's instructions. To approve the bioinformatics results, the extracted RNA was reverse-transcribed into cDNA by using the PrimeScript RT Reagent Kit (TaKaRa, China), and then detect the fold changes of miRNAs by using the qRT-PCR. The qRT-PCR response setting procedures were as follows: $3 \mathrm{~min}$ for $95^{\circ} \mathrm{C}, 40$ cycles at $\left(95^{\circ} \mathrm{C}\right.$ for $5 \mathrm{~s}, 58^{\circ} \mathrm{C}$ for $\left.35 \mathrm{~s}\right), 72^{\circ} \mathrm{C}$ for $\left.60 \mathrm{~s}\right), 65^{\circ} \mathrm{C}$ for $5 \mathrm{~s}, 95^{\circ} \mathrm{C}$ for $0.5 \mathrm{~s}$. All experiments were conducted more than three times. The expression levels of miRNAs were defined based on the cycle threshold (Ct), and relative expression levels were calculated using the 2- $\Delta \Delta C$ t method. The expression levels of U6 was used as reference miRNA. The primers used for the experimental validation are listed in Table 1.

\subsection{Cell culture}

Human TNBC cell line (MDA-MB-231) was preserved in our laboratory. MDA-MB-231 cells were cultured in DMEM medium (Saimike, Carlsbad, CA, USA) with $10 \%$ fetal bovine serum, $100 \mathrm{U} / \mathrm{mL}$ penicillin and $100 \mathrm{mg} / \mathrm{mL}$ streptomycin, and with a humidified incubator containing $5 \% \mathrm{CO} 2$ at $37^{\circ} \mathrm{C}$.

\subsection{Cell transfection}

Hsa-miR-3616-3p mimic, inhibitor or a matched miRNA negative control (NC) were purchased from GenePharma (Shanghai, China). Cell transfections were conducted with Lipofectamine 2000 (Invitrogen, Carlsbad, CA, USA) in accordance with the manufacturer's protocols. qRT-PCR was used to measure transfection efficiency.

\subsection{Wound healing assays}


Cell migration was mensurated by wound healing assays. MDA-MB-231 cells were transfected with mimic NC or hsa-miR-3616-3p mimic and inhibitor NC or hsa-miR-3616-3p inhibitor, and when the cells reached $90 \%$ confluence, the monolayer of the cells was scratched with the tip of the 200 microliter pipette to produce wound stripes. Cells migration into the wound was observed through five randomly selected microscopic horizons at two predetermined time points $(0$ and $24 \mathrm{~h})$, respectively. The area of the remaining gaps after cell growth after 0 and $24 \mathrm{~h}$ intervals were measured, and the area of the gap in NC group was set to $0 \mathrm{~h}$ as $100 \%$ and relative cell migration was calculated. A larger gap is considered to be lower cell migration. All experiments were conducted at least three times.

\subsection{Transwell assays}

Transwell assays were performed to evaluate cell migration and invasion capability. Transfected MDA-MB-231 cells were starved in serum-free medium for two hours, detached, and resuspended in medium containing serum-free. For migration assays, $1.5 \times$ TNBC cells were suspended in $200 \mu \mathrm{L}$ serum-free medium and inoculated into the upper chambers (BD BioCoat, MA, USA), and then $500 \mu \mathrm{L}$ complete medium was added into the bottom chambers. For invasion assays, $3 \times$ TNBC cells were suspended in $200 \mu \mathrm{L}$ serum-free medium and inoculated into the upper chambers coated with matrigel (BD Biosciences, NJ, USA), and then $500 \mu \mathrm{L}$ complete medium was added into the bottom chambers. After $24 \mathrm{~h}$, the cells on the upper chambers were removed and cells on the lower compartment were fixed with ethanol and stained by crystal violet, then photographed and counted with a microscope (Leica, Wetzlar, Germany).

\subsection{GO and KEGG enrichment analysis of target genes of hsa-miR-3616-3p}

The DAVID (https://david.ncifcrf.gov/) is a database that provides systematic and comprehensive annotation information of biological functions for genes and proteins [18]. Gene ontology (GO) is an international standard classifification system of gene function[19]. It includes biological process (BP), cellular component (CC), and molecular function (MF). Kyoto Encyclopedia of Genes and Genomes (KEGG) is a database for systematic analysis of gene function and genomic information[20]. We used DAVID to carry out GO functional annotation and KEGG pathway enrichment analysis on the target genes of hsa-miR-3616-3p, $p<0.05$ were considered statistically significant[21].

\subsection{Bioinformatics analysis of transcription factors of hsa-miR-3616-3p}

The transcription factors (TFs) of hsa-miR-3616-3p were found and analyzed by the Transmir database (http://www.cuilab.cn/transmir). The function of TFs were conducted by KEGG enrichment analysis. The expression of TFs in normal tissues and TNBC tissue samples from TCGA were analyzed by the Ualcan database (http://ualcan.path.uab.edu/).

\subsection{Statistical analysis}

Statistical analyses were performed in GraphPad Prism 8.0 software. The values of different groups were represented by the mean \pm SD. The comparison of expression levels of TNBC and non-TNBC tumor tissues were analyzed by paired $t$ test. $p<0.05$ was considered to represent a statistically significant difference.

\section{Results}

\subsection{Identification of differentially expressed Non-coding RNAs}

To explore the role of systems biology in the pathogenesis of BC, we analyzed two chip data of GSE57897 and GSE97811 by GEO2R. They were normalized by Limma rapid differential analysis tool in sanger box (http://sangerbox.com/) to eliminate batch effects. Based on adjust $p$ value $<0.05$ and $\mid \log 2$ Fold Changel $\geq 1$ were used as the cut-off criteria, the differentially expressed miRNAs were derived 962, included 943 upregulated miRNAs and 19 downregulated miRNAs between breast tissue samples and normal breast tissue samples (Figure 1A). We selected 4 candidate miRNAs for further analysis: top2 up-regulated miRNAs as hsa-miR-4428 and hsa-miR-575, and top2 down-regulated miRNAs as hsa-miR-3616-3p and hsa-miR-3909 (Figure 1B and Table 2).

\subsection{OS analysis of candidate miRNAs}

OS is considered to be the best end point in tumor clinical trials. When the survival time of patients can be fully evaluated, it is the first end point. The prognostic value of 4 candidate miRNAs was measured by the online Kaplan-Meier Plotter survival analysis server in three subtypes of BC, namely Luminal (positive for ER and/or PR), Her-2 overexpression (negative for ER and PR, positive for Her-2), and TNBC. Obtain OS of patients based on the expression of 4 candidate miRNAs in breast cancer and its three subtypes. Based on the screen criteria $p<0.05$, OS analysis of 4 candidate miRNAs in BC patients and luminal subtype of $\mathrm{BC}$ patients, the results showed the patients 
with high expression of 4 candidate miRNAs were related to a poor prognosis (Figure 2A and Figure 2B). As for Her-2 overexpression subtype, the results showed that the high expression of hsa-miR- 575 was related to a poor prognosis, and 3 candidate miRNAs (hsa-miR4428, hsa-miR-3909 and hsa-miR-3616-3p) were not associated with OS ( $p>0.05$ ) (Figure 2C). In TNBC subtype, the low expression of 4 candidate miRNAs exhibited shorter OS time than those with high expression (Figure 2D). Highly aggressive TNBC was considered to have less favorable prognosis compared to other BC types. Thus, we further explored the relationship between 4 candidate miRNAs and TNBC.

\subsection{Validation of candidate miRNAs by qRT-PCR}

To further confirm the expression level of 4 candidate miRNAs in TNBC, we conducted qRT-PCR on 15 TNBC tissues and 15 non-TNBC tissues. Compared to non-TNBC group, our results showed that the expressions of hsa-miR-4428 ( $p=0.0371$, Figure $3 \mathrm{~A})$ and hsa-miR3616-3p ( $p=0.0346$, Figure 3D) were significantly decreased in TNBC tissues, the expressions of hsa-miR-575 ( $p=0.0059$, Figure 3B) was significantly increased in TNBC tissues but hsa-miR-3909 was no obvious statistical differences in TNBC tissues $(p=0.7897$, Figure 3C). However, the qRT-PCR results of hsa-miR-575 and hsa-miR-3616-3p were consistent with the result of microarray data (Figure 3E). Moreover, hsa-miR-3616-3p was the most significant and stable down-regulation, and the low hsa-miR-3616-3p expression was related to a poor prognosis in TNBC. All of the above results suggested that hsa-miR-3616-3p may be correlated with TNBC. Therefore, we focused on hsa-miR-3616-3p for further study.

\subsection{Down-regulation of hsa-miR-3616-3p enhances migration and invasion in TNBC cells}

To further explore whether the expression of hsa-miR-3616-3p could regulate cell migration and invasion in TNBC, MDA-MB-231 cells were transfected with mimic NC or hsa-miR-3616-3p mimic, inhibitor NC or hsa-miR-3616-3p inhibitor, respectively. The transfection efficiency was measured by qRT-PCR (Figure 4A). Next, wound healing assays and transwell assays were performed to examine the effect of hsa-miR-3616-3p with migration and invasion in MDA-MB-231 cell (Figure 4B - 4D). All of the above results showed that up-regulation of hsa-miR-3616-3p inhibited the migratory and invasive capabilities of MDA-MB-231 cells, while down-regulation of hsa-miR-3616-3p revealed an opposite effect. These data suggested that hsa-miR-3616-3p should exert an anti-oncogenic role in the TNBC, and the downregulation of hsa-miR-3616-3p induced migratory and invasive in TNBC cells.

\subsection{GO and KEGG Enrichment Analysis of Target genes of hsa-miR-3616-3p}

In order to better understand the biological function of hsa-miR-3616-3p, we found 4,218 target genes of hsa-miR-3616-3p by using the TargetScan database (http://www.targetscan.org/) and chosed 1654 target genes based on the criteria: Cumulative weighted context++ score $<-2$. Next, the functions of 1654 target genes were performed by GO and KEGG enrichment analysis. GO results showed that the target genes of hsa-miR-3616-3p signifificantly enriched in protein binding, integral component of plasma membrane and transcription factor activity, and so on (Figure5A). Moreover, KEGG analysis showed that the target genes of hsa-miR-3616-3p were enriched in focal adhesion, cell adhesion molecules (CAMs) and regulation of action cytoskeleton, and so on (Figure5B).

\subsection{Bioinformatics Analysis of transcription factors of hsa-miR-3616-3p}

Transcription factors (TFs) could bind to specific sequences of DNA to exert transcription effect on one or more genes. The dysregulated miRNAs were controlled by transcription factors. By using the Transmir database (http://www.cuilab.cn/transmir), we found that 24 TFs were predicted to bind on the promoter domain of hsa-miR-3616-3p (Figure 6A). KEGG enrichment analysis of 24 TFs was operated by DAVID and the results showed that these TFs of hsa-miR-3616-3p were enriched in transcriptional misregulation in cancer, TGF-beta signaling pathway and pathways in cancer, and so on (Figure 6B). Moreover, the Transmir database revealed that 7 of the 24 TFs were associated with the breast tissues (Table 3). We validated and visualized the the expression of 7 TFs in Ualcan database (http://ualcan.path.uab.edu/) with TNBC tissue samples from TCGA. The results confirmed that ERG, MYC and NRF1 were downregulated $(p<0.05)$ and GRHL2 and KDM5B were upregulated $(p<0.05)$ in TNBC tissues compared with normal tissues, and CTCF and TRIM25 were no statistically significant $(p>0.05)$ in TNBC tissues compared with normal tissues (Figure 6C-6l). These data demonstrated that hsa-miR3616-3p could be regulated by these TFs (ERG, MYC,NRF1,GRHL2 and KDM5B) through activating or inhibiting promoter domain.

\section{Discussion}

Worldwide, breast cancer is the most common cancer affecting women, and its incidence and mortality rates are expected to increase significantly the next years[22]. This highlights the pressing need for new biomarkers and novel mechanism for BC prediction and therapy. More and more studies have confirmed that miRNAs have already been the new biomarkers for diagnosis, prognosis, therapy 
prediction and therapeutic tools for breast cancer[23-25]. Meanwhile, miRNAs are key regulators in biological processes of BC, such as the occurrence and development of $\mathrm{BC}$, proliferation signal transduction, resistance to cell death, activation of cell migration and invasion[26-28]. However, only a few miRNAs have been well characterized and little is known about the biological functions of miRNAs in BC. Excitingly, the high-throughput technology, public databases and bioinformatics methods greatly promote the understanding of miRNAs in BC. In this sduty, integrating multiple sets of BC miRNA microarrays to explore key miRNAs via bioinformatics analysis and public databases is a promising approach to identify the potential miRNA and provide new biomarkers for BC.

In our study, we analyzed two chip data from GSE57897 and GSE97811, and harvested 962 differentially expressed miRNAs between BC tumor and normal breast tissue samples, including 943 up-regulated miRNAs and 19 down-regulated miRNAs. The top 2 most significantly up-regulated miRNAs (hsa-miR-4428 and hsa-miR-575) and down-regulated miRNAs (hsa-miR-3909 and hsa-miR-3616-3p) were chosed to further experiments. Most of them have been reported that were associated with other cancers and diseases. Silencing of hsa-miR-4428 weaken the proliferative and migratory abilities of lung adenocarcinoma cells[29]. Hsa-miR-575 targeted BLID to promote growth and invasion of non-small cell lung cancer cells[30], moreover hsa-miR-575 exerted oncogenic function in hepatocellular carcinoma by increasing hepatocellular carcinoma proliferation and metastasis[31]. Downregulation of hsa-miR-3909 enhanced the inflammation level in the progression of rheumatic heart disease by acting on it's target gene IL1R1 [32]. However, hsa-miR-3616-3p hasn't been reported. Overall, it suggests that these 4 miRNAs in BC should be the important molecules.

Gene expression profiling studies have shown that breast cancer is not caused by a single gene. Molecular subtyping of breast cancer can provide important prognostic information and provide a reference for the choice of treatment strategies[33]. We have mainly explored OS analysis of the 4 candidated miRNAs in the luminal, Her-2 overexpression and TNBC subtypes. TNBC constitutes the most aggressive molecular subtype among BC, usually appears in the form of high-grade invasive ductal carcinoma, with a higher rate of early recurrences, distant metastases, and poorer prognosis compared to other BC subtypes[34]. Our results showed that high expression of 4 candidated miRNAs had a worse prognosis in both BC and luminal subtype. In Her-2 overexpression subtype, the OS of hsa-miR-575 was only statistically significant, the main reason may be that the number of Her-2 overexpression samples was relatively small. In contrast, low expression of 4 candidated miRNAs had a worse prognosis in TNBC subtype. We further focused on the relationship between 4 candidated miRNAs and TNBC. Subsequently, we tested the expression of 4 candidated miRNAs by qRT-PCR on 15 TNBC and 15 nonTNBC tissue samples and found that the expression level of hsa-miR-3616-3p in TNBC tissue samples was consistent and stable with the microarray data. Further, it was showed that the down-expression hsa-miR-3616-3p could enhanced the metastasis and invasion ability of TNBC cells by wounding heal assays and transwell assays. In a word, hsa-miR-3616-3p would be a new diagnostic and prognostic marker or therapeutical target for TNBC patients.

MicroRNA (miRNAs) are small non-coding RNA (ncRNA) molecules that bind messenger RNA (mRNA) complementary sequences and generally direct post-transcriptional silencing in the 3'-untranslated region (UTR) of target genes[35]. To understand the relationship between hsa-miR-3616-3p and target genes, we screened out 1654 target genes based on the predictions and analyses of TargetScan database, and then we conducted functions of 1654 target genes by GO and KEGG enrichment analyses. The function of these target genes were enriched in focal adhesion, cell adhesion molecules (CAMs) and regulation of action cytoskeleton, and so on. Studies had shown that high focal adhesion kinase expression in breast carcinoma was associated with triple-negative phenotype [36] and focal adhesion kinase (FAK) inhibition prevented the migration of TNBC[37]. Previous researches had indicated that cell adhesion was an important pathway leading to TNBC poor prognosis[38,39]. And regulation of action cytoskeleton inhibited migration, invasion and adhesion of TNBC cells[40]. These results suggested that hsa-miR-3616-3p may bind target gene to affect biological behavior of TNBC via the above enrichment pathways. However, the target genes of hsa-miR-3616-3p were showing up in greater numbers, and it dose need further research to understand.

Studies had indicated that the expression of miRNA was regulated by transcription factors[41, 42], so we predicted and analyzed transcription factors of hsa-miR-3616-3p by Transmir database. Transmir is a database of regulatory relationships between transcription factors and miRNA collated from the literature[43]. We found 24 transcription factors were related with hsa-miR-3616-3p, and the results of KEGG enrichment analysis of 24 TFs showed that these TFs of hsa-miR-3616-3p were enriched in transcriptional misregulation in cancer, TGF-beta signaling pathway and pathways in cancer, and so on, which were related to occurrence and development of BC. Moreover, 7 of the 24 TFs were associated with the breast tissues. The expression of these TFs was evaluated by Ualcan database. In TNBC tissues compared with normal tissues, the expression of ERG, MYC and NRF1 were downregulated ( $p<0.05)$, GRHL2 and KDM5B were upregulated $(p<0.05)$. However, CTCF and TRIM25 were no statistically significant $(p>0.05)$.. Studies had shown that high expressed ERG was relevant to prostate cancer including invasion and metastasis, EMT, epigenetic reprogramming, differentiation and inflammation[44]. MYC, high expressor in TNBC, which could inhibited miR-200b to promote the epithelial to mesenchymal transition and 
mammosphere formation of TNBC cells[45]. High expressed NRF1 in BC, showed unfavorable prognosis with a high risk of breast cancer death in white women[46]. It was reported that high GRHL2 expression was highly correlated with worse survival in TNBC, with regulation of EMT[47]. In previous studies, high expressed KDM5B enhanced TNBC cells metastasis and progression by regulating hsa-miR-448[48]. Together, it is possible that the expression of hsa-miR-3616-3p may be regulated by transcription factors.

The above conclusions indicated that target genes and TFs may regulate hsa-miR-3616-3p via these pathways affecting the progression of TNBC, which were worth further study. Together, unreported hsa-miR-3616-3p may play a key role in TNBC migration and invasion and may provide new ideas for the diagnosis, prognosis, and therapeutic targeting of TNBC.

\section{Conclusion}

In this study, based on database and bioinformatical analysis, we found out that down-expressed hsa-miR-3616-3p have a worse prognosis in TNBC. Moreover, down-expressed hsa-miR-3616-3p regulated the metastasis and invasion ability of TNBC cells by wounding heal assays and transwell assays. These results suggest that hsa-miR-3616-3p may be potential biomarker to diagnosis, prognosis, and therapy of TNBC.

\section{Declarations}

\section{Acknowledgements}

Not applicable.

\section{Authors' contributions}

$\mathrm{KW}$ and $\mathrm{YS}$ conceived and designed the research, contributed experiment methods and analysis tool. KW performed the experiments and drafted the paper, YS and ZZ collected. ZZ, RP and HL analyzed the data. All authors read and support the final manuscript .

\section{Funding}

This study was supported by the National Natural Science Foundation of China (grant numbers 81700639).

\section{Availability of data and materials}

The datasets used and/or analyzed during the current study are available from the corresponding author on reasonable request.

\section{Ethics approval and consent to participate}

The study was conducted in accordance with the Helsinki Declaration and has been approved by the Ethics Committee of Chongqing Medical University.Signed written informed consents were obtained from the patients and/or guardiants.

\section{Consent for publication}

Not applicable.

\section{Competing interests}

The authors declare that there is no conflict of interest regarding the publication

of this paper.

\section{Author details}

${ }^{1}$ Department of Cell Biology and Genetics, Chongqing Medical University, Chongqing 400016, China

${ }^{2}$ Department of Bioinformatics, Chongqing Medical University, Chongqing 400016, China

\section{References}

1. DeSantis, C., et al., Breast cancer statistics, 2013. CA Cancer J Clin, 2014. 64(1): p. 52-62. 
2. Gupta, I., et al., Triple Negative Breast Cancer Profile, from Gene to microRNA, in Relation to Ethnicity. Cancers (Basel), 2019. 11(3).

3. William J. Gradishar MD Betsy Bramsen Professor of Breast Oncology Director, M.D.C.f.W.s.C.C., Triple-Negative Breast Cancer. Northwestern University Feinberg School of Medicine, 2012.

4. Sharma, P., Biology and Management of Patients With Triple-Negative Breast Cancer. Oncologist, 2016. 21(9): p. 1050-62.

5. Hwang, S.Y., S. Park, and Y. Kwon, Recent therapeutic trends and promising targets in triple negative breast cancer. Pharmacol Ther, 2019. 199: p. 30-57.

6. Guestini, F., et al., Triple negative breast cancer chemosensitivity and chemoresistance: current advances in biomarkers indentification. Expert Opin Ther Targets, 2016. 20(6): p. 705-20.

7. Tiwari, A., B. Mukherjee, and M. Dixit, MicroRNA Key to Angiogenesis Regulation: MiRNA Biology and Therapy. Curr Cancer Drug Targets, 2018. 18(3): p. 266-277.

8. Zhen, N., et al., CirchMGCS1 Promotes Hepatoblastoma Cell Proliferation by Regulating the IGF Signaling Pathway and Glutaminolysis. Theranostics, 2019. 9(3): p. 900-919.

9. Li, X.N., et al., RNA sequencing reveals the expression profiles of circRNA and indicates that circDDX17 acts as a tumor suppressor in colorectal cancer. J Exp Clin Cancer Res, 2018. 37(1): p. 325.

10. Gonzales, J.C., et al., Comparison of circulating MicroRNA 141 to circulating tumor cells, lactate dehydrogenase, and prostatespecific antigen for determining treatment response in patients with metastatic prostate cancer. Clin Genitourin Cancer, 2011. 9(1): $\mathrm{p}$. 39-45.

11. Du, Y., et al., MicroRNA-141 inhibits migration of gastric cancer by targeting zinc finger E-box-binding homeobox 2. Mol Med Rep, 2015. 12(3): p. 3416-3422.

12. Hu, M., et al., MicroRNA-141 regulates Smad interacting protein 1 (SIP1) and inhibits migration and invasion of colorectal cancer cells. Dig Dis Sci, 2010. 55(8): p. 2365-72.

13. Huang, Y., et al., miR-141 regulates TGF-beta1-induced epithelial-mesenchymal transition through repression of HIPK2 expression in renal tubular epithelial cells. Int J Mol Med, 2015. 35(2): p. 311-8.

14. Li Lin1t, H.L., Yanbo Wang2t, Xiaomao Yin1, Yanwei Hu1, Jinlan Huang1, Tingyu Ren1, Hui Xu3, Lei Zheng1* and Xi Chen2*, microRNA-141 inhibits cell proliferation and invasion and promotes apoptosis by targeting hepatocyte nuclear factor-3 $\beta$ in hepatocellular carcinoma cells. BMC Cancer, 2014.

15. Li, P., et al., Downregulation of miRNA-141 in breast cancer cells is associated with cell migration and invasion: involvement of ANP32E targeting. Cancer Med, 2017. 6(3): p. 662-672.

16. Lv, M., et al., miR141-CXCL 1-CXCR2 signaling-induced Treg recruitment regulates metastases and survival of non-small cell lung cancer. Mol Cancer Ther, 2014. 13(12): p. 3152-62.

17. Clough, E. and T. Barrett, The Gene Expression Omnibus Database. Methods Mol Biol, 2016. 1418: p. 93-110.

18. Huang, D.W., et al., DAVID Bioinformatics Resources: expanded annotation database and novel algorithms to better extract biology from large gene lists. Nucleic Acids Res, 2007. 35(Web Server issue): p. W169-75.

19. Thomas, P.D., The Gene Ontology and the Meaning of Biological Function. Methods Mol Biol, 2017. 1446: p. 15-24.

20. Research, M.K.a.S.G.A., KEGG: Kyoto Encyclopedia of Genes and Genomes. Nucleic Acids Research, 2000.

21. Jiang, W.D. and Z.H. Ye, Integrated analysis of a competing endogenous RNA network in renal cell carcinoma using bioinformatics tools. Biosci Rep, 2019. 39(7).

22. Anastasiadi, Z., et al., Breast cancer in young women: an overview. Updates Surg, 2017. 69(3): p. 313-317.

23. Bertoli, G., C. Cava, and I. Castiglioni, MicroRNAs: New Biomarkers for Diagnosis, Prognosis, Therapy Prediction and Therapeutic Tools for Breast Cancer. Theranostics, 2015. 5(10): p. 1122-43.

24. Ding, L., et al., MicroRNAs Involved in Carcinogenesis, Prognosis, Therapeutic Resistance and Applications in Human Triple-Negative Breast Cancer. Cells, 2019. 8(12).

25. Nassar, F.J., R. Nasr, and R. Talhouk, MicroRNAs as biomarkers for early breast cancer diagnosis, prognosis and therapy prediction. Pharmacol Ther, 2017. 172: p. 34-49.

26. Yang, S.S., et al., Breast cancer-derived exosomes regulate cell invasion and metastasis in breast cancer via miR-146a to activate cancer associated fibroblasts in tumor microenvironment. Exp Cell Res, 2020. 391(2): p. 111983.

27. Wang, Z., et al., Blockade of miR-3614 maturation by IGF2BP3 increases TRIM25 expression and promotes breast cancer cell proliferation. EBioMedicine, 2019. 41: p. 357-369. 
28. Kim, E.S., et al., C-reactive protein binds to integrin alpha2 and Fcgamma receptor l, leading to breast cell adhesion and breast cancer progression. Oncogene, 2018. 37(1): p. 28-38.

29. Ying, K., et al., ACTA2-AS1 suppresses lung adenocarcinoma progression via sequestering miR-378a-3p and miR-4428 to elevate SOX7 expression. Cell Biol Int, 2020. 44(12): p. 2438-2449.

30. Wang, H., et al., MicroRNA-575 targets BLID to promote growth and invasion of non-small cell lung cancer cells. FEBS Lett, 2015. 589(7): p. 805-11.

31. Wang, Y.N., et al., MicroRNA-575 regulates development of gastric cancer by targeting PTEN. Biomed Pharmacother, 2019. 113: p. 108716.

32. Lu, Q., et al., Comprehensive microRNA profiling reveals potential augmentation of the IL 1 pathway in rheumatic heart valve disease. BMC Cardiovasc Disord, 2018. 18(1): p. 53.

33. Therese Sørliea, b., c, Charles M. Peroua,d, Robert Tibshiranie, Turid Aasf, Stephanie Geislerg, Hilde Johnsenb, Trevor Hastiee, Michael B. Eisenh, Matt van de Rijni, Stefanie S. Jeffreyj, Thor Thorsenk, Hanne Quistl, John C. Matesec, Patrick O. Brownm, David Botsteinc, Per Eystein Lønningg, and Anne-Lise Børresen-Daleb,n, Gene expression patterns of breast carcinomas distinguish tumor subclasses with clinical implications. 2001.

34. Vagia, E., D. Mahalingam, and M. Cristofanilli, The Landscape of Targeted Therapies in TNBC. Cancers (Basel), 2020. 12(4).

35. Jacinta-Fernandes, A., et al., Allele-specific miRNA-binding analysis identifies candidate target genes for breast cancer risk. NPJ Genom Med, 2020. 5: p. 4.

36. Vita M Golubovskaya1*, L.Y., Austin Miller3, Melissa Hughes1, Jason Wilson1, David Wang4, Elizabeth Brese3, Wiam Bshara3, Stephen Edge1, Carl Morrison3 and William G Cance1*, High focal adhesion kinase expression in breast carcinoma is associated with lymphovascular invasion and triple-negative phenotype. 2014.

37. Rigiracciolo, D.C., et al., Focal adhesion kinase (FAK) activation by estrogens involves GPER in triple-negative breast cancer cells. J Exp Clin Cancer Res, 2019. 38(1): p. 58.

38. Cox, O.T., et al., PDLIM2 Is a Marker of Adhesion and beta-Catenin Activity in Triple-Negative Breast Cancer. Cancer Res, 2019. 79(10): p. 2619-2633.

39. Cai, Y.C., et al., ANGPTL4 overexpression inhibits tumor cell adhesion and migration and predicts favorable prognosis of triplenegative breast cancer. BMC Cancer, 2020. 20(1): p. 878.

40. Graminha, A.E., et al., A novel ruthenium(ii) gallic acid complex disrupts the actin cytoskeleton and inhibits migration, invasion and adhesion of triple negative breast tumor cells. Dalton Trans, 2021. 50(1): p. 323-335.

41. Martinez, N.J. and A.J. Walhout, The interplay between transcription factors and microRNAs in genome-scale regulatory networks. Bioessays, 2009. 31(4): p. 435-45.

42. Martinez, N.J., et al., A C. elegans genome-scale microRNA network contains composite feedback motifs with high flux capacity. Genes Dev, 2008. 22(18): p. 2535-49.

43. Tong, Z., et al., TransmiR v2.0: an updated transcription factor-microRNA regulation database. Nucleic Acids Res, 2019. 47(D1): p. D253-D258.

44. Adamo, P. and M.R. Ladomery, The oncogene ERG: a key factor in prostate cancer. Oncogene, 2016. 35(4): p. 403-14.

45. Pang, Y., et al., MYC and DNMT3A-mediated DNA methylation represses microRNA-200b in triple negative breast cancer. J Cell Mol Med, 2018. 22(12): p. 6262-6274.

46. Ramos, J., et al., Sensitivity to differential NRF1 gene signatures contributes to breast cancer disparities. J Cancer Res Clin Oncol, 2020. 146(11): p. 2777-2815.

47. Mooney, S.M., et al., The GRHL2/ZEB Feedback Loop-A Key Axis in the Regulation of EMT in Breast Cancer. J Cell Biochem, 2017. 118(9): p. 2559-2570.

48. Bamodu, O.A., et al., Aberrant KDM5B expression promotes aggressive breast cancer through MALAT1 overexpression and downregulation of hsa-miR-448. BMC Cancer, 2016. 16: p. 160.

\section{Tables}

Table1: Primers used for real-time PCR 


\begin{tabular}{|lll|}
\hline MiRNA symbol & Primer category & Primer sequence \\
\hline Hsa-miRNA-4428 & Forward primer & CGCAAGGAGACGGGAACA \\
\cline { 2 - 3 } & Reverse primer & AGTGCAGGGTCCGAGGTATT \\
\cline { 2 - 3 } & Reverse transcriptase & GTCGTATCCAGTGCAGGGTCCGAGGTATTCGCACTGGATACGACGCTCCA \\
\hline Hsa-miRNA-3909 & Forward primer & CGCGGAGCCAGTTGGAC \\
\cline { 2 - 3 } & Reverse primer & AGTGCAGGGTCCGAGGTATT \\
\cline { 2 - 3 } & Reverse transcriptase & GTCGTATCCAGTGCAGGGTCCGAGGTATTCGCACTGGATACGACGCTCCT \\
\cline { 2 - 3 } & Reverse primer & AGTGCAGGGTCCGAGGTATT \\
\cline { 2 - 3 } Hsa-miRNA-3616-3p & Reverse transcriptase & GTCGTATCCAGTGCAGGGTCCGAGGTATTCGCACTGGATACGACAGACTG \\
\cline { 2 - 3 } & Forward primer & CGCGAGGGCATTTCATGAT \\
\cline { 2 - 3 } & Reverse primer & AGTGCAGGGTCCGAGGTATT \\
\cline { 2 - 3 } & Reverse transcriptase & GTCGTATCCAGTGCAGGGTCCGAGGTATTCGCACTGGATACGACGCCTGC \\
& Forward primer & CTCGCTTCGGCAGCACA \\
\cline { 2 - 3 } & Reverse primer & AACGCTTCACGAATTTGCGT \\
\cline { 2 - 3 } & &
\end{tabular}

Table2:Characteristics of candidate MiRNAs

\begin{tabular}{|llllll|}
\hline MiRNA symbol & RNAcentral ID & Location(chr) & MiRNA sequence & \multicolumn{2}{l|}{$\begin{array}{l}\text { CHIP-seq datas } \\
\text { adj.P.Value }\end{array}$} \\
& & & & $\begin{array}{l}\text { log2Fold } \\
\text { Change }\end{array}$ \\
\hline Hsa-miRNA-4428 & URS000075CBFD & $\begin{array}{l}1: 237,471,164- \\
237,471,185\end{array}$ & UGUCCUCUAGGGCCUGCAGUCU & $1.04 E-43$ & 5.42019 \\
\hline Hsa-miRNA-575 & URS000075C5FB & $4: 82,753,352-82,753,370$ & CGAGGGCAUUUCAUGAUGCAGGC & $3.69 E-29$ & 4.66718 \\
\hline Hsa-miRNA-3909 & URS000022B729 & $\begin{array}{l}22: 35,335,710- \\
35,335,731\end{array}$ & CAAGGAGACGGGAACAUGGAGC & $3.32 E-07$ & -1.92102 \\
\hline $\begin{array}{l}\text { Hsa-miRNA-3616- } \\
\text { 3p }\end{array}$ & URS000075D1FC & $\begin{array}{l}20: 47,167,026- \\
47,167,048\end{array}$ & GAGCCAGUUGGACAGGAGC & $2.73 E-08$ & -1.67422 \\
\hline
\end{tabular}

Table3ロ Characteristics of transcription factor of hsa-miRNA-3616 


\begin{tabular}{|c|c|c|c|c|c|}
\hline $\begin{array}{l}\text { TF } \\
\text { name }\end{array}$ & Ensembel gene id & Location(chr) & Transcription start site & Binding site & Evidence \\
\hline \multirow[t]{2}{*}{ CTCF } & ENSG00000102974 & $\begin{array}{l}\text { Chr16:67,562,467- } \\
67,639,177\end{array}$ & chr20: 46894843 & $\begin{array}{l}\text { chr20:46894648- } \\
46894887(\text { score }=312)\end{array}$ & $\begin{array}{l}\text { CHIP- } \\
\text { seq+experiments }\end{array}$ \\
\hline & & & & $\begin{array}{l}\text { chr20:46894830- } \\
46894926(\text { score }=384)\end{array}$ & $\begin{array}{l}\text { CHIP- } \\
\text { seq+experiments }\end{array}$ \\
\hline ERG & ENSG00000157554 & $\begin{array}{l}\text { Chr21:38,380,027- } \\
38,661,780\end{array}$ & chr20: 46894843 & $\begin{array}{l}\text { chr20:46894602- } \\
46894838(\text { score }=469)\end{array}$ & $\begin{array}{l}\text { CHIP- } \\
\text { seq+experiments }\end{array}$ \\
\hline \multirow[t]{6}{*}{ GRHL2 } & ENSG00000083307 & $\begin{array}{l}\text { Chr8:101,492,439- } \\
101,669,726\end{array}$ & chr20:46894624(NM_005244) & $\begin{array}{l}\text { chr20:46894203- } \\
\text { 46894548(score }=1000)\end{array}$ & CHIP-seq \\
\hline & & & & $\begin{array}{l}\text { chr20:46894205- } \\
46894540(\text { score }=1000)\end{array}$ & CHIP-seq \\
\hline & & & & $\begin{array}{l}\text { chr20:46894212- } \\
\text { 46894488(score }=1000)\end{array}$ & CHIP-seq \\
\hline & & & chr20:46894624(NM_172110) & $\begin{array}{l}\text { chr20:46894203- } \\
\text { 46894548(score=1000) }\end{array}$ & CHIP-seq \\
\hline & & & & $\begin{array}{l}\text { chr20:46894205- } \\
\text { 46894540(score=1000) }\end{array}$ & CHIP-seq \\
\hline & & & & $\begin{array}{l}\text { chr20:46894212- } \\
\text { 46894488(score }=1000)\end{array}$ & CHIP-seq \\
\hline \multirow[t]{3}{*}{ KDM5B } & ENSG00000117139 & $\begin{array}{l}\text { Chr1:202,724,495- } \\
\text { 202,808,487 }\end{array}$ & chr20: 46894843 & $\begin{array}{l}\text { chr20: 46894786- } \\
\text { 46894903(score=322) }\end{array}$ & $\begin{array}{l}\text { CHIP- } \\
\text { seq+experiments }\end{array}$ \\
\hline & & & chr20:46894624(NM_005244) & $\begin{array}{l}\text { chr20: } 46894536- \\
46894706(\text { score }=456)\end{array}$ & CHIP-seq \\
\hline & & & chr20:46894624(NM_172110) & $\begin{array}{l}\text { chr20: } 46894536- \\
46894706(\text { score }=456)\end{array}$ & CHIP-seq \\
\hline \multirow[t]{2}{*}{ MYC } & ENSG00000136997 & $\begin{array}{l}\text { Chr8:127,735,434- } \\
127,742,951\end{array}$ & chr20: 46894843 & $\begin{array}{l}\text { chr20: } 46894622- \\
46894728(\text { score }=371)\end{array}$ & $\begin{array}{l}\text { CHIP- } \\
\text { seq+experiments }\end{array}$ \\
\hline & & & & $\begin{array}{l}\text { chr20: } 46894823- \\
46894924(\text { score }=553)\end{array}$ & $\begin{array}{l}\text { CHIP- } \\
\text { seq+experiments }\end{array}$ \\
\hline \multirow[t]{2}{*}{ NRF1 } & ENSG00000106459 & $\begin{array}{l}\text { Chr7:129,611,720- } \\
129,757,082\end{array}$ & chr20: 46894843 & $\begin{array}{l}\text { chr20: } 46894733- \\
\text { 46894903(score=554) }\end{array}$ & $\begin{array}{l}\text { CHIP- } \\
\text { seq+experiments }\end{array}$ \\
\hline & & & & $\begin{array}{l}\text { chr20: } 46894754- \\
46894879 \text { (score }=362 \text { ) }\end{array}$ & $\begin{array}{l}\text { CHIP- } \\
\text { seq+experiments }\end{array}$ \\
\hline \multirow[t]{5}{*}{ TRIM25 } & ENSG00000121060 & $\begin{array}{l}\text { Chr17: } \\
56,836,387- \\
56,914,080\end{array}$ & chr20: 46894843 & $\begin{array}{l}\text { chr20: } 46894628- \\
46894820(\text { score }=268)\end{array}$ & $\begin{array}{l}\text { CHIP- } \\
\text { seq+experiments }\end{array}$ \\
\hline & & & chr20:46894624(NM_005244) & $\begin{array}{l}\text { chr20: } 46894629- \\
46895110(\text { score }=537)\end{array}$ & CHIP-seq \\
\hline & & & & $\begin{array}{l}\text { chr20: } 46894892- \\
46895078(\text { score }=360)\end{array}$ & CHIP-seq \\
\hline & & & chr20:46894624(NM_172110) & $\begin{array}{l}\text { chr20: } 46894629- \\
46895110 \text { (score }=537 \text { ) }\end{array}$ & CHIP-seq \\
\hline & & & & $\begin{array}{l}\text { chr20: } 46894892- \\
46895078(\text { score }=360)\end{array}$ & CHIP-seq \\
\hline
\end{tabular}

\section{Figures}



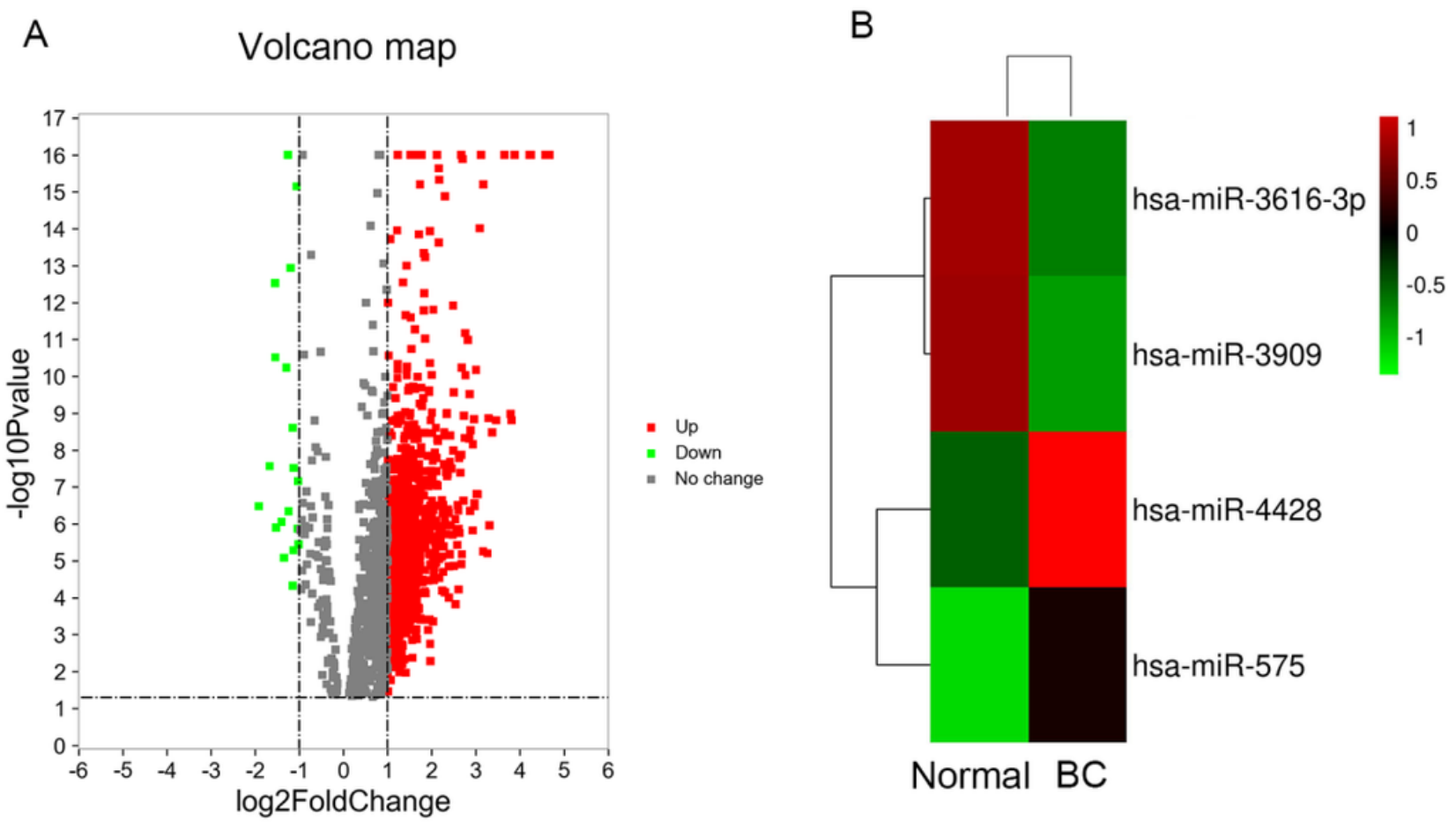

Normal BC

Figure 1

Volcano plot demonstrating the differential expression of miRNAs and four candidate miRNAs (A) Red points mean up-regulated miRNAs screened on the basis $\mid \log 2$ fold change| $\geq 1$ and adjust $p$ value $<0.05$. Green points mean down-regulated miRNAs screened on the basis $\mid \log 2$ fold changel $\geq 1$ and adjust $p$ value $<0.05$. Black points represent genes with no difference in expression. (B) The heatmap of 4 candidate miRNAs. 


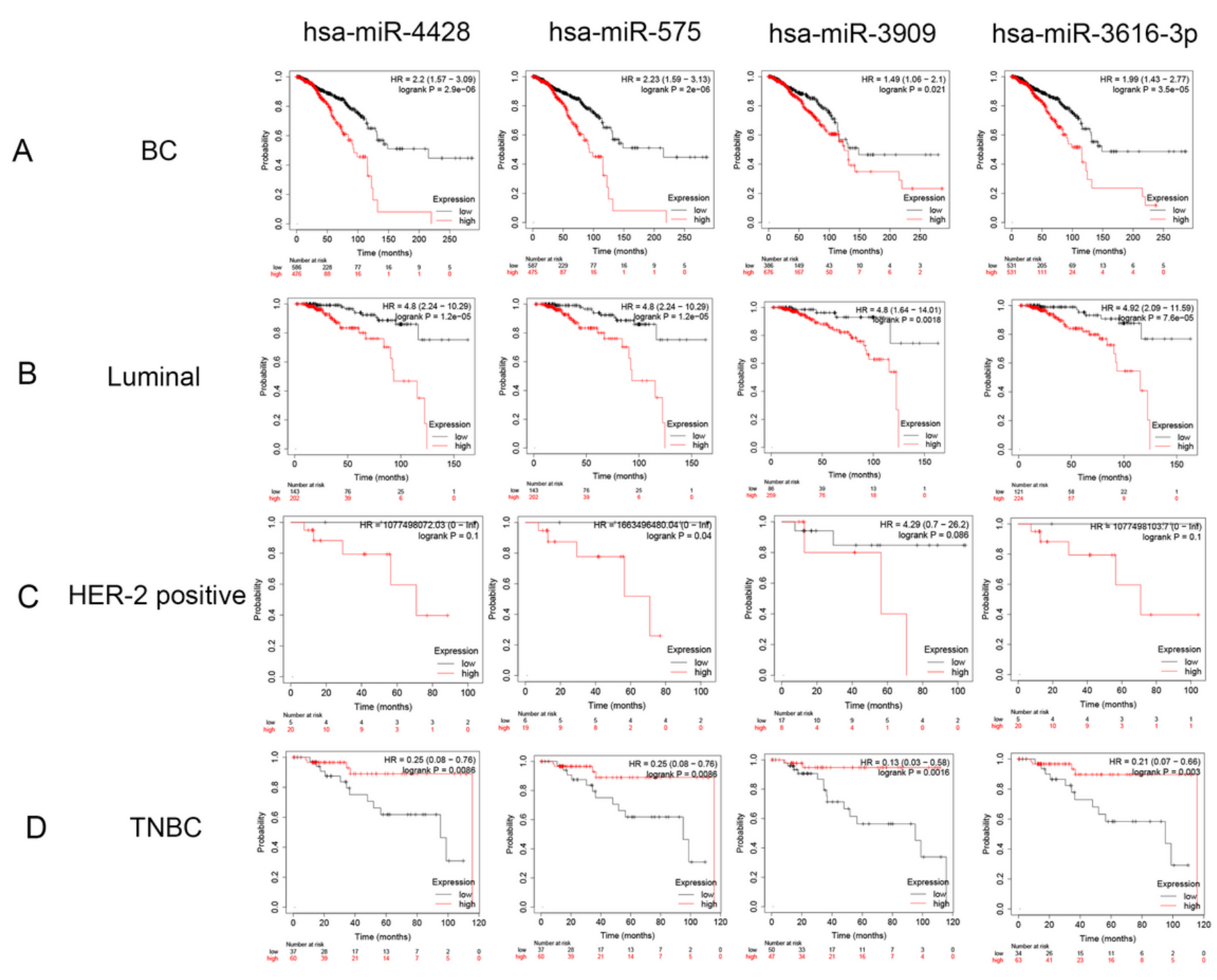

\section{Figure 2}

OS analysis of four candidate miRNAs in BC and three subtypes of BC patients using Kaplan-Meier Plotter (A) OS analysis of 4 candidate miRNAs in BC patients. The results showed the patients with high expression of 4 candidate miRNAs were related to a poor prognosis. $(\mathrm{p}<0.05)(B)$ OS analysis of 4 candidate miRNAs in Luminal subtype (positive for ER and/or PR) of BC patients. The results showed the patients with high expression of 4 candidate miRNAs were related to a poor prognosis. ( $>0.05)$ (C) OS analysis of 4 candidate miRNAs in a Her-2 overexpression subtype(negative for ER and PR, positive for Her-2) of BC patients. The results showed the patients with the high expression of hsa-miR-575 was related to a poor prognosis, and 3 candidate miRNAs (hsa-miR-4428, hsa-miR-3909 and hsa-miR-3616$3 p$ ) were not associated with OS. ( $p>0.05$ ) (D) OS analysis of 4 candidate miRNAs in TNBC patients. The results showed the patients with low expression of 4 candidate miRNAs were related to a poor prognosis. $(p<0.05) p<0.05$ was considered to indicate a statistically significant difference. 
A
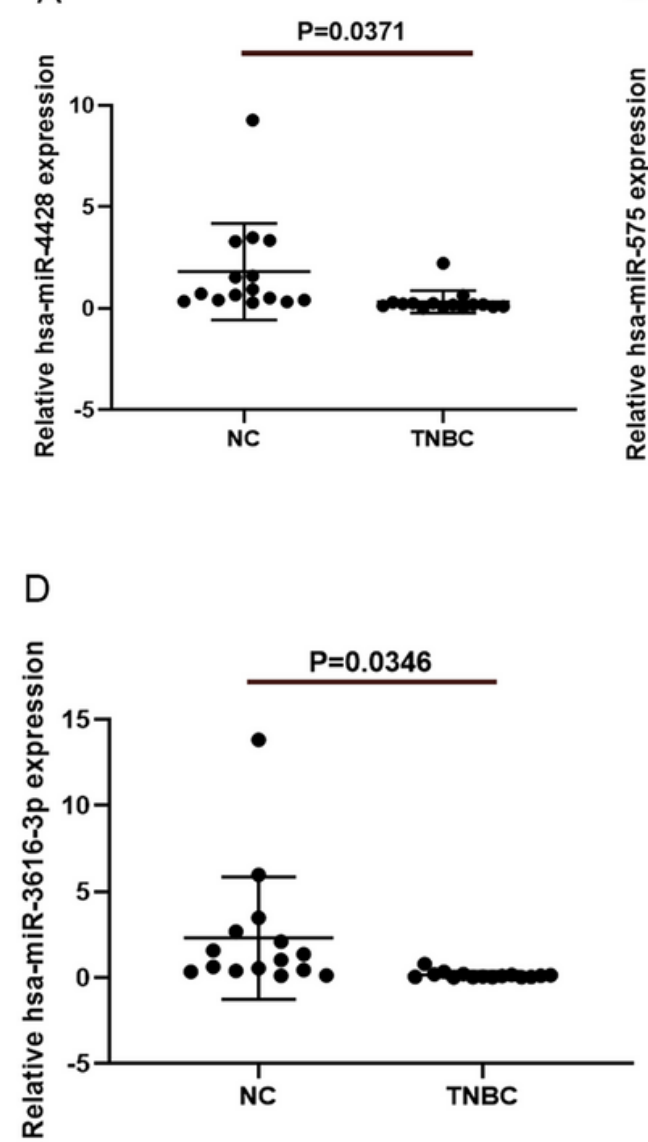

B

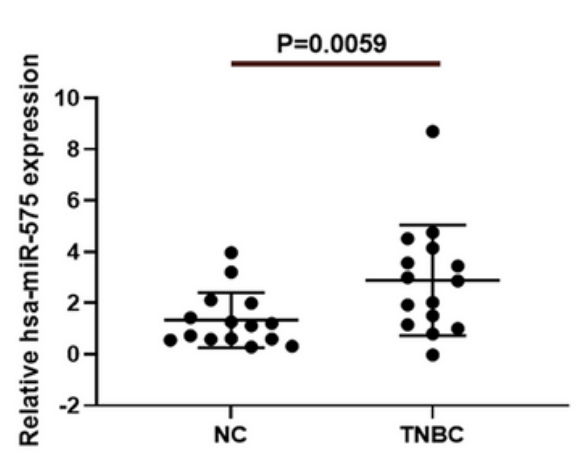

E
C
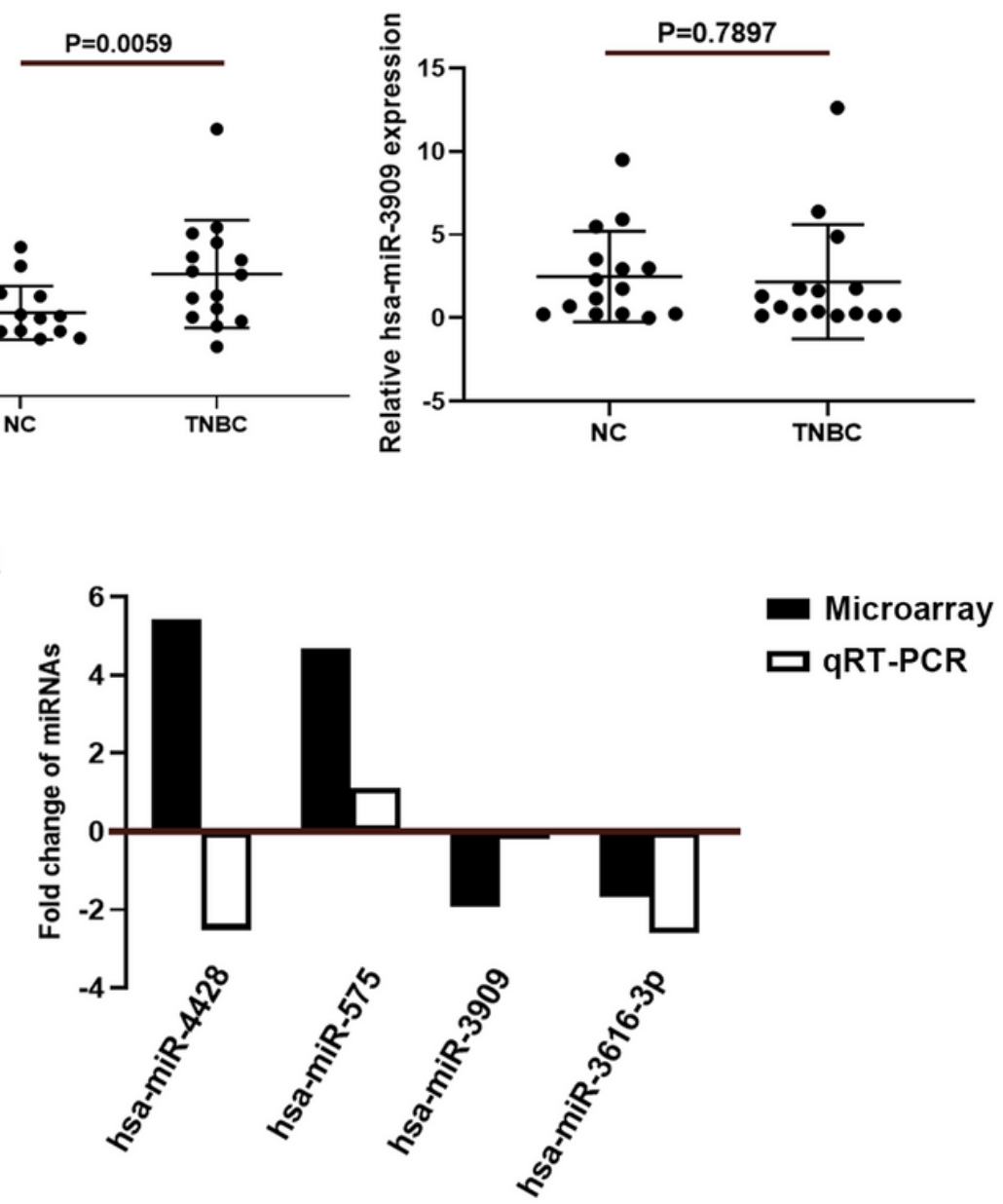

Figure 3

Validation of four candidate miRNAs through qRT-PCR (A) The relative expression of hsa-miR-4428 was lower in TNBC tissues than that in non-TNBC tissues. $(p=0.0371)(B)$ The relative expression of hsa-miR- 575 was higher in TNBC tissues than that in non-TNBC tissues. $(p=0.0059)(C)$ The relative expression of hsa-miR-3909 was lower in TNBC tissues than that in non-TNBC tissues. $(p=0.7897)(D)$ The relative expression of hsa-miR-3616-3p was lower in TNBC tissues than that in non-TNBC tissues. $(p=0.0346)$ (E) Comparison of the expression of 4 candidate miRNAs identified by microarray and qRT-PCR. Our qRT-PCR datas showed that the expressions of hsa-miR575, hsa-miR-3909 and hsa-miR-3616-3p were consistent with the microarray data. 


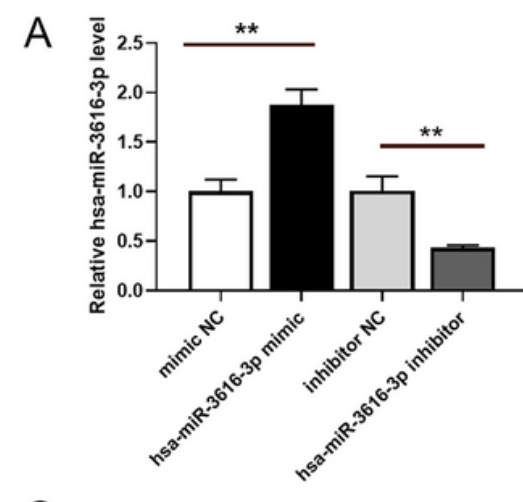

C

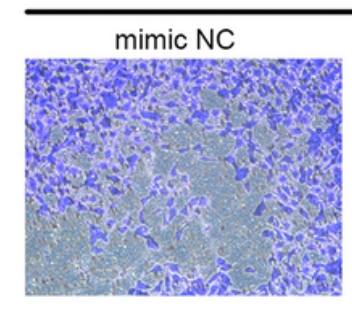

D

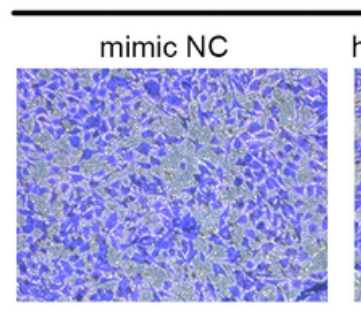

B
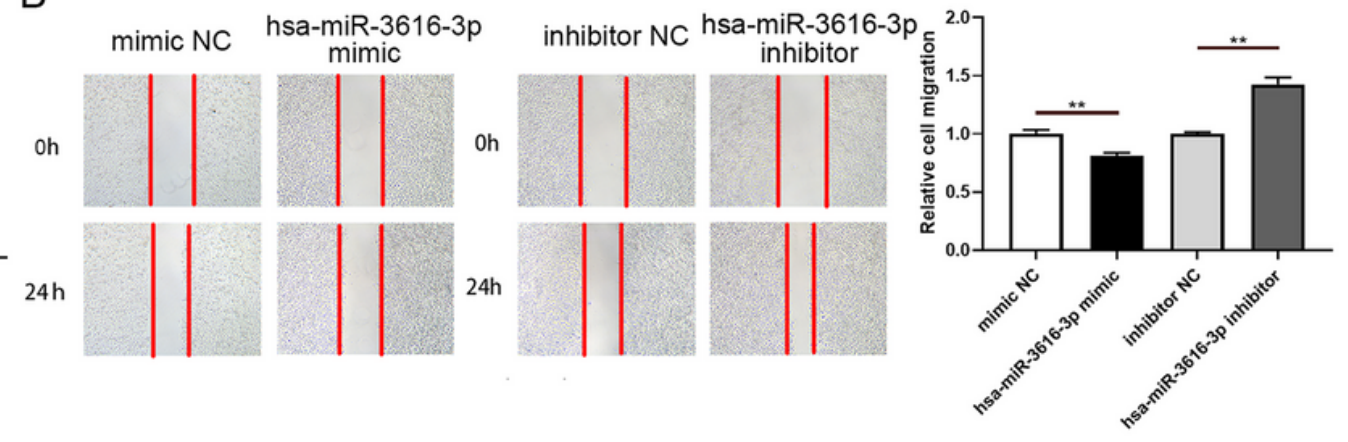

migration
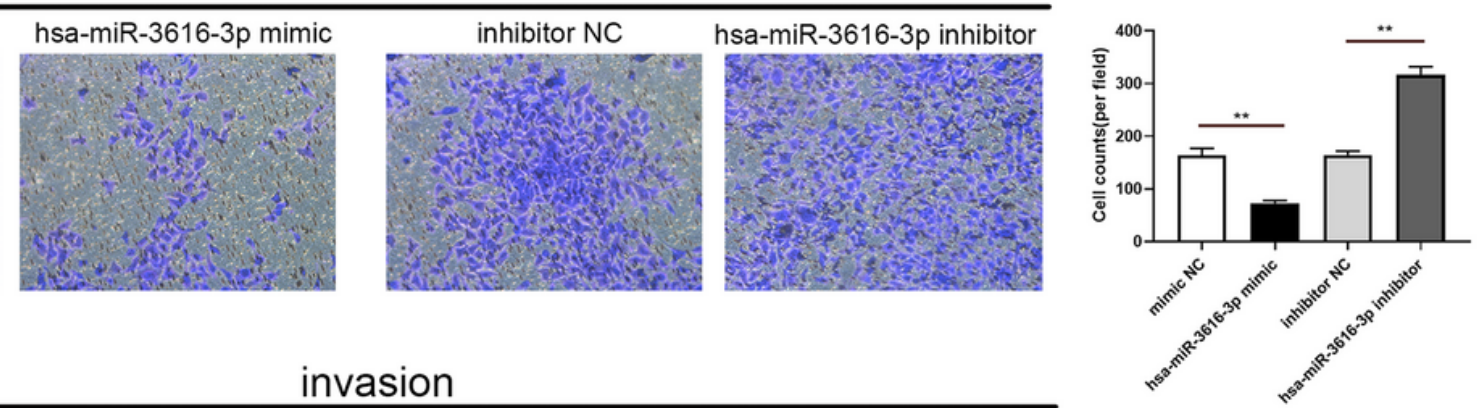

invasion
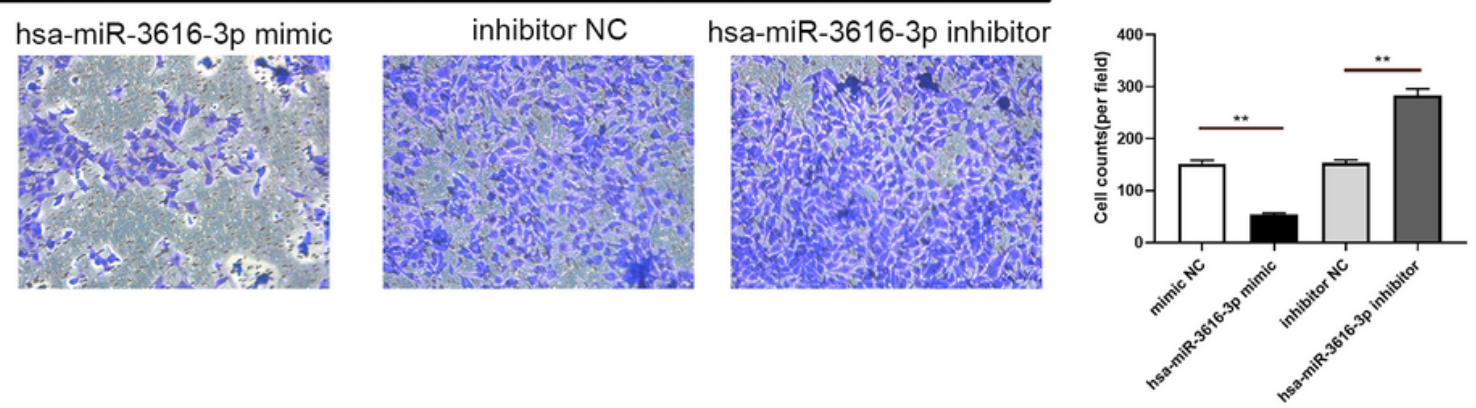

\section{Figure 4}

Effect of hsa-miR-3616-3p overexpression on TNBC cell migration and invasion (A) qRT-PCR analysis of hsa-miR-3616-3p expression in TNBC cells transfected with mimic NC or hsa-miR-3616-3p mimic;inhibitor NC or hsa-miR-3616-3p inhibitor. (B) Cell migration capacities were detected by wound healing assays after transfection (magnification, $\times 50$ ). (C) Cell migration abilities were determined by transwell migration assays after transfection (magnification, $\times 100$ ). (D) Cell invasion abilities were determined by transwell invasion assays after transfection (magnification, $\times 100$ ) (Data were showed as mean $\pm S D,{ }^{*}$ means $p<0.05,{ }^{* \star}$ means $p<0.01, * \star \star$ means $p<0.001$ ) 
A

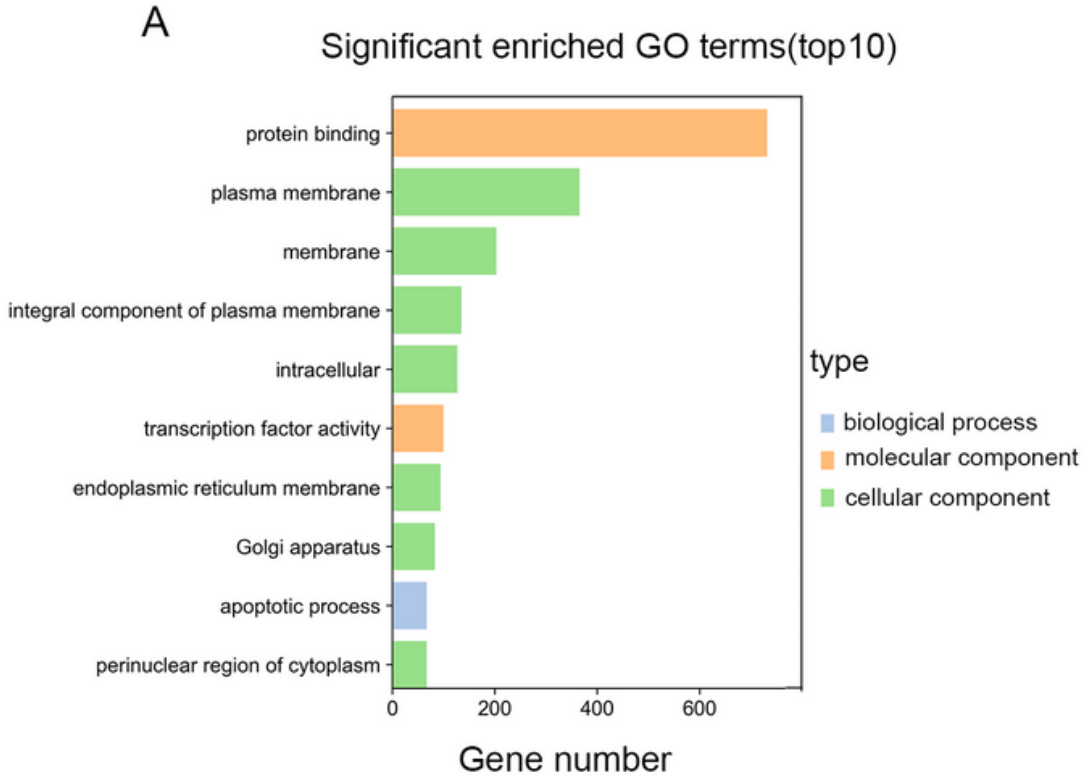

B

Significant enriched KEGG pathways(top10)

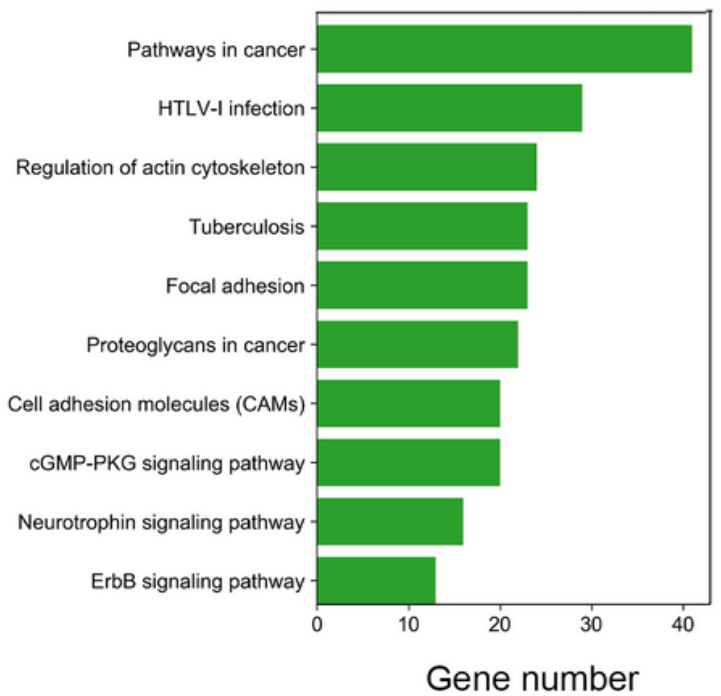

\section{Figure 5}

GO and KEGG Enrichment Analysis of Target genes of hsa-miR-3616-3p (A) Top 10 significantly enriched GO annotations of target genes of hsa-miR-3616-3p, including three groups (molecular function, biological process and cellular component). ( $p<0.05)$ (B) Top 10 functional pathways of target genes of hsa-miR-3616-3p through KEGG analysis. $(p<0.05)$ 

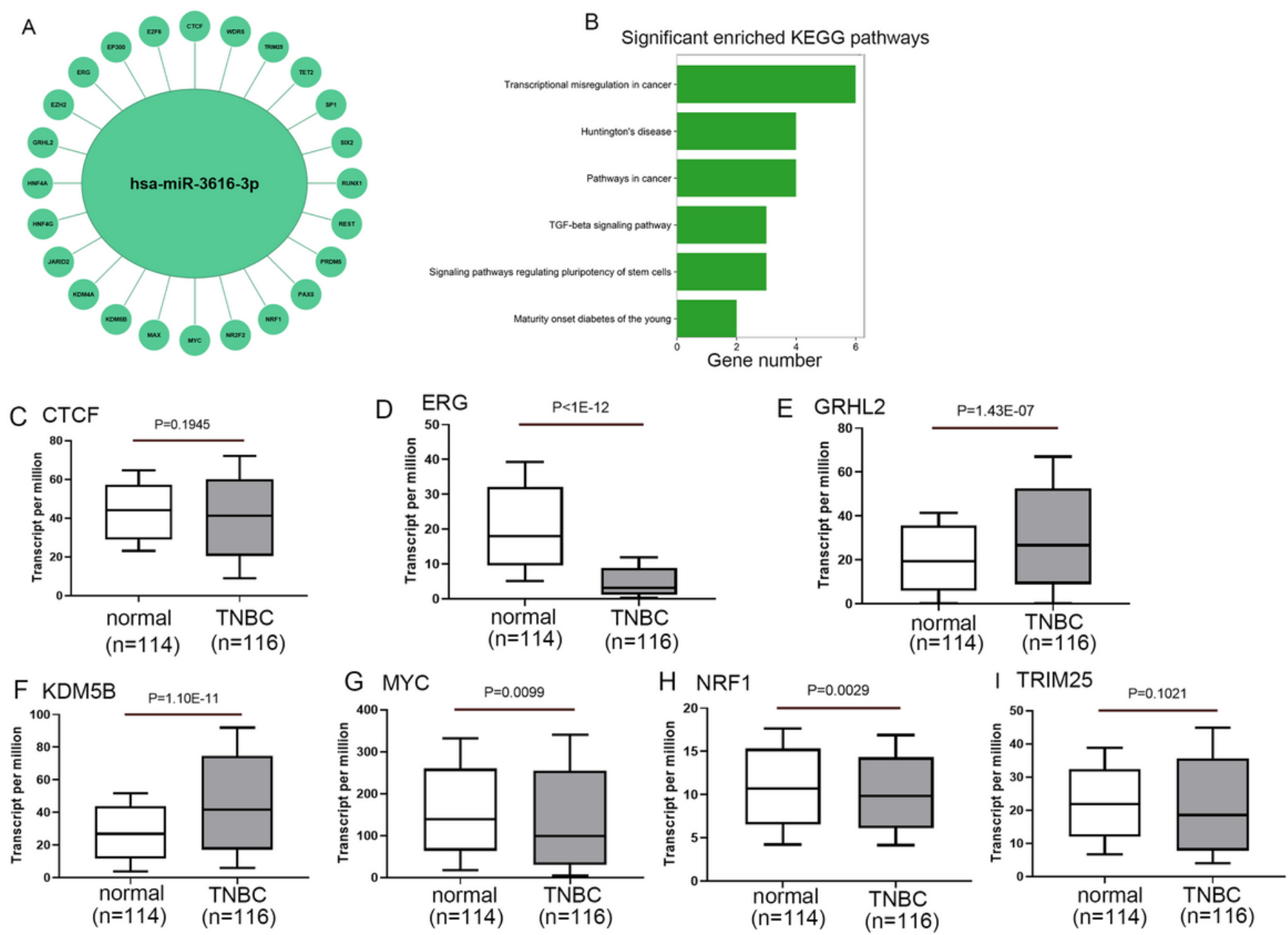

Figure 6

GO and KEGG Enrichment Analysis of transcription factors of hsa-miR-3616-3p (A) 24 TFs were predicted to bind on the promoter domain of hsa-miR-3616-3p (B) Functional pathways of transcription factors of hsa-miR-3616-3p through KEGG analysis. (p<0.05) (C) $-(I)$ ERG, MYC and NRF1 were downregulated $(p<0.05)$ and GRHL2 and KDM5B were upregulated $(p<0.05)$ in TNBC tissues compared with normal tissues. CTCF and TRIM25 were no statistically significant ( $p>0.05)$ in TNBC tissues compared with normal tissues. 